\title{
Fault Diagnosis in Dynamic Systems Using Recurrent Neural Networks
}

\author{
M.A.FKIRIN \\ Dept. of Industrial and Control \\ Engineering, Faculty of \\ Electronic Engineering, \\ Menofia University, Egypt.
}

\author{
RANIA.ELHAG \\ Dept. of Industrial and Control \\ Engineering, Faculty of \\ Electronic Engineering, \\ Menofia University, Egypt.
}

\begin{abstract}
The aim of this paper is to discuss the methodology of Fault Detection and Diagnosis (FDD) in dynamic systems. Fault diagnosis is characterized as a control system that controls the ability to adapt system component faults automatically. Fault detection is an implementation of using the error signals, where when error signal is zero or approximately zero. A coupled water tank system was used as a study case model for implementing and testing the proposed methodology. The developed system should generate a set of signals to notify the process operator about the faults that are occurring, enabling changes in control strategy or control parameters. Due to the damage risks involved with sensors, actuators and structural faults of the real plant, the data set of the faults are computationally generated and the results will be collected from numerical simulations of the process model. A Recurrent Neural Networks (RNN) is employed in this paper for modeling the used system. This paper shows how to determine the structure and how to estimate the results using the gradient-based algorithm which it is allowed to obtain a Neural Network with relatively small modeling uncertainly. It describes how to increase the stability of dynamic systems using FDD based on RNN. Faults in the study case are faults in the sensors, faults in the actuators and the structural faults. The faults which are presented in the controlled system are reduced to $98 \%$ from the default value.
\end{abstract}

Keyword:Fault Detection and Diagnosis (FDD), Recurrent Neural Networks (ANN), Level control system for two coupled tanks.

\section{Introduction}

Since most of the industrial systems deployed everywhere in our daily live can't work efficiently without faults leading to the collapse of the system. For this reason the need of reliable diagnose is very important to monitor system performance, especially for complex modern technological systems. In modern life, many industrial systems rely on automatic control to stabilize the process. For this reason, traditional control has been created quite over the past six decades. While the conventional controller has a primary terminal objective of framework stability and performance with all parts working normally, there is a broad requirement for control systems to actually work in a project. Most traditional control design methods do not consider possible framework component faults cases. A control system is designed using these systems can perform well in a typical process, but can fail as systems even if a secondary component is defective. In this way, taking into consideration the ultimate objective of enhancing the robustness of the framework against component faults. For this reason new plan should be prepared to detect faults in dynamic systems with highly accurate. This is Artificial Neural Networks (ANN) to diagnose faults in dynamic systems. The fault can be described as an unbalanced deviation at least a characteristic or parameter of the accepted system [1], [2].

We discuss a level control in two coupled tank system to diagnose faults which are appeared. The system consists of two basic stages. The first stage is the design of the system for identifying and isolating the faults to determine the component by which the faults in actuators or sensors in the system through mathematical model of the system (LUENBURGER Observer) using Artificial Neural Network. In the second stage, the system is activated to overcome the faults detected in the first stage to deal with these faults and overcome them so that the performance of the system with the kidneys faults is the same performance without the presence [3].

In recent years, a Neural Networks (NN) has been widely applied in the field of chemical engineering, in process control, and as a powerful tool of function approximation and pattern recognition. NN also are studied and applied to Fault Detection and Diagnosis problem. NN have been used predictor or dynamic models for fault diagnosis, the fault diagnosis and pattern classifiers for fault identification. NN have been successfully applied in modeling dynamic systems as well as Fault Detection and Diagnostics. NN are better than classic methods because they can handle the most complex systems and Neural Networks provide an excellent mathematical tool for dealing with non-linear systems with great flexibility. $\mathrm{NN}$ are also robust with respect to incorrect or missing data. Protecting relaying based on Artificial Neural Networks is not affected by a change in system operating conditions. NN have high computation rate, large input error tolerance. In general, Artificial Neural Networks can be applied to fault diagnosis in order to solve both modeling and classification problems [4], [5]. The proposed algorithm is implemented using MATLAB running on an Intel ${ }^{\circledR}$ Core $^{\mathrm{TM}}$ i5 CPU platform 
with 2GB RAM running Windows ${ }^{\mathrm{TM}}$ 7. Several simulation experiments have been conducted to evaluate performance of the algorithm and validate the functionality of the proposed technique which used to tune the parameters of the model.

Fault Detection and Diagnosis using Artificial Neural Networks with multilayer percepteron (MLP) were introduced by (Diago Leite Reboucas, Fabio Meneghetti Ugulino de Araujo and Andre Laurindo Maitelli, 2012). MLP have a large structure which make evaluation very slow. The paper uses Artificial Neural Networks that include in its structure a recurrent connection, namely the Artificial Neural Networks with Local Recurrent Structure (ANN-LRS). Artificial Neural Networks must have a minimal structure in order to allow for a fast evaluation, so the recurrent structure is required. . The training algorithm used was the Levenberg-Marquardt (LMA), available in mathematical software MATLAB math program. It is an important mathematical tool for improving the accuracy of predictions in data mining. Artificial Neural Networks use back propagation as a learning algorithm to compute a gradient descent with respect to weights.

\section{Fault Detection and Diagnosis}

Fault Detection and Diagnosis plan (FDD) is highly sensitive to faults, which is robust to the uncertainty models and classes of working conditions and external disturbances, and has the ability to give accurate and most detailed data about the system at the earliest opportunity after the fault event to be suited before more damage to the system or loss of service happens. According to Chiang et al. (2001), among other functions, these systems can detect, diagnose and eliminate faults, ensuring that the process operations satisfy the performance specifications.

Additionally, the information provided by a monitoring system should not only inform the system operator about what is going on, but also help him to take corrective actions in order to remedy the problem. As a result, the ineffective time will be reduced, the system protection will be increased and the operational costs will be decreased. Chiang et al. (2001) shows that there are four states involved in the process monitoring: fault detection, fault isolation, fault diagnosis and fault recovery, as shown in Fig. 1. Although arranged as a sequence of actions, all states are not always strictly necessary. Often, automated changes from one state to another is transparent to the operator, displaying only the crucial information to take appropriate action.[1],[6],[16].

\section{Fault Classification}

According to Garter, the faults classification is based on location in the physical system, classification is based on mathematical properties, based on the time behavior characteristics [7]. Faults and failures are classified based on their location. According to their location, faults are classified into actuators, component and sensor faults. Actuators faults are faults that corrupt the actuating signal, i.e. they modify the influence of the controller on the system by partial or total (complete) loss of control action [8]. Total actuator faults can occur as a result of burned or cut wiring breakage, short circuits or due to a foreign body presented in the actuator. Partially failed actuators, which produce only part of the normal actuation, result from pneumatic or hydraulic leakage; fall in the supply voltage, or increased resistance. Since actuators are frequently considered as the entrance to the controlled framework, actuator faults serious outcomes on the framework execution.

Actuator faults have been one of the imperative viewpoints and active research area in the most recent decades [9], [10], [11], [12]. It is generally very difficult to add extra hardware redundancy of actuators to increase reliability. Because they are expensive, large in size and need a large driving signals. Sensors are used in the control system to measure and convert the physical quantities of interest into a signal. A fault in the sensor means an incorrect measurement from the sensor, which in turn can result in a continuous constant offset as compared to the true value. Sensor faults can degrade the feedback system performance even in the presence of a wall-designed controller. Therefore it is important to detect and isolate sensor faults in early time. These faults can be divided into partial or total faults. Total sensor faults give information that is not the real value of the measured physical parameter. Partial sensor faults give readings in such a way that some useful information could be received.

To increase fault tolerance, and due to their smaller sizes, sensors can be duplicated in the system. These are faults in components of the plant itself, and represent changes in the system's physical parameters such as mass, aerodynamic coefficients, or damping constant, which are often due to structural damages. They are very diverse and cover an extensive class of unanticipated situations. Computer systems can be described by five basic characteristics: functionality, ease of use, performance, cost and reliability. The term dependability refers to the ability of the system to provides a service that can be, justifiably, reliable. Based on this logic, a reliable system is divided into the three parts according to Avizienis et al. (2000), as shown in figure 2.

The first group is used to provide analysis on the quality of a reliable system. In the second group, the term failure should be used to indicate when a deviation of the behavior of the system occurs. However, there is an error related to the status of the system and can lead to failure. The term error is caused by an error and is associated with a defect. Typically, an error can be defined as a defect that has the potential to generate errors. So, fault is the deviation of at least one characteristic of variable of the system from acceptable/ usual behavior, but failure permanent interruption of the system's ability to perform a specific function under specified operating conditions [13].

\section{Level Control Process}

We present the development of FDD system in a dynamic process. The process involved consists of a two coupled tank are connected in such away to create a nonlinear control system of structural constraints in fig. 3. The equipment is fully controlled by MATLAB math program and all of the sensors and actuators are driven by signals generated inside MATLAB. 


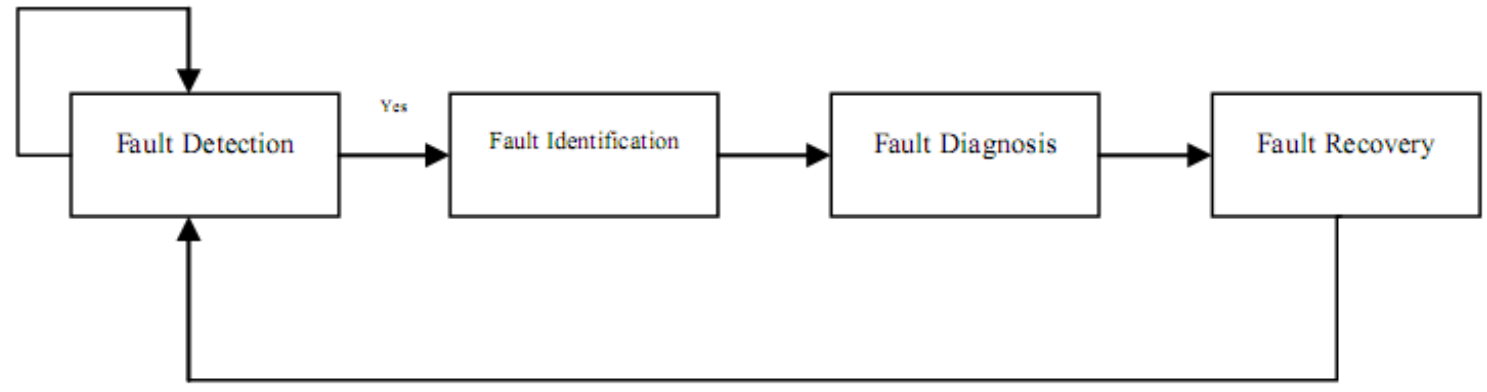

Fig. 1 States of fault detection and diagnosis, based on Chiang et al. (2001).

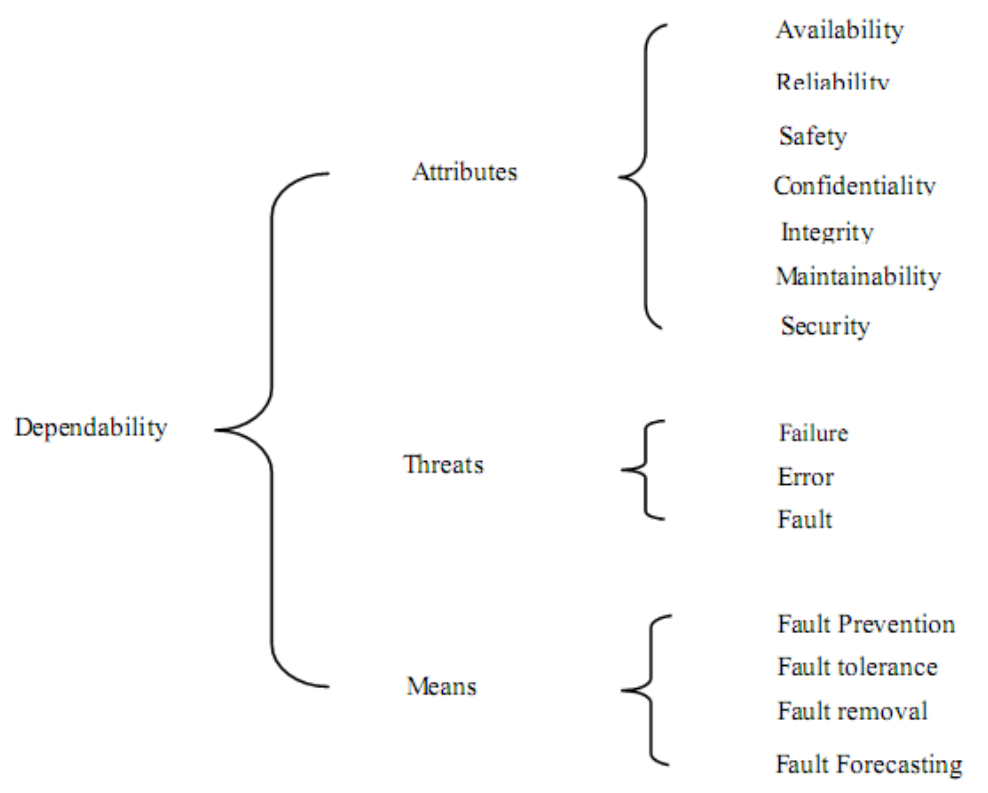

Fig. 2 Dependability classification, based on Avizienis et al. (2000).

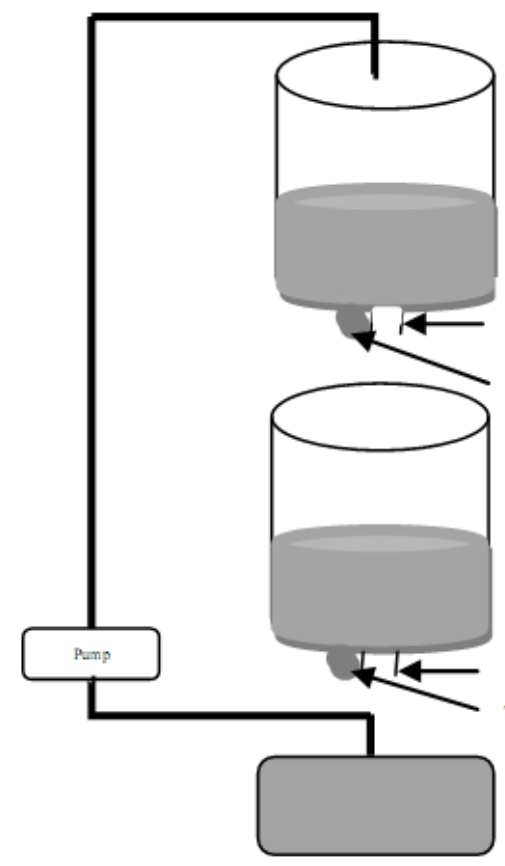

(a)

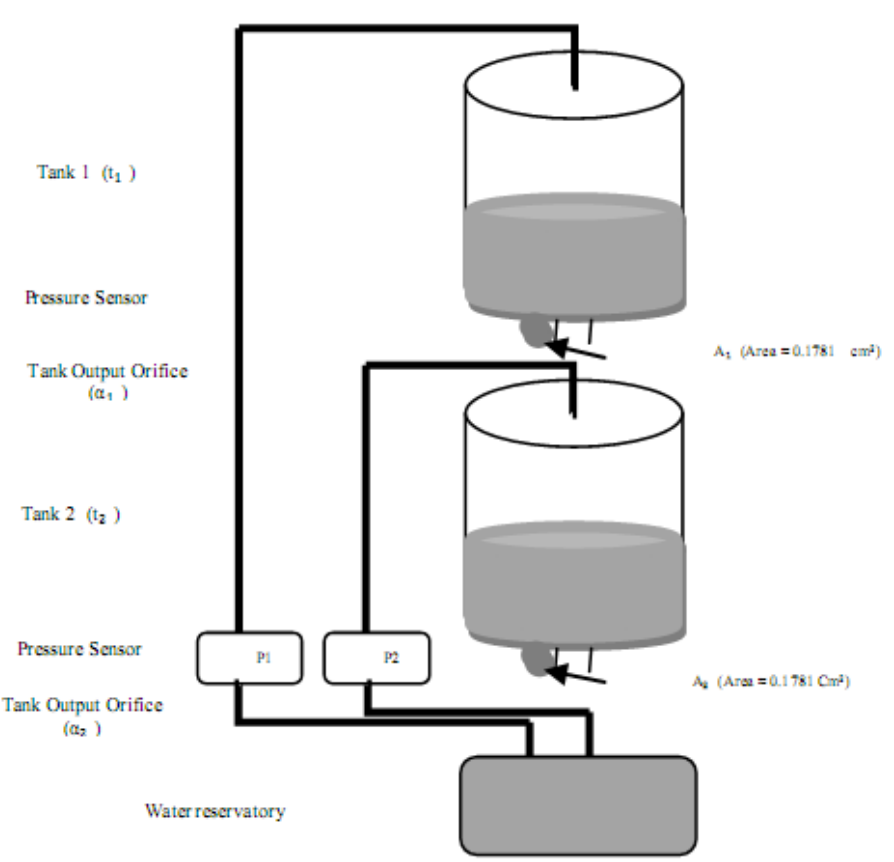

(b) Proposed Configuration

Fig. 3 Case study - Coupled Water Tanks. 
Tanks $\left(t_{1}, t_{2}\right)$ are mounted on the front of the support base and placed in such a way that the water flows from the upper tank $t_{1}$ to the lower reservoir $t_{2}$ through $\alpha_{1}$ and from $t_{2}$ through $\alpha_{2}$. The resulting water flow varies according to the orifices $\alpha_{1}$ and $\alpha_{2}$, which are available in three different diameters. Since the two tanks have the same cross-sectional area $\left(A_{1}=A_{2}=A\right)$.

Mathematical model that describes the dynamics of these tanks is not that simple, because the general equations of motion and energy that describe fluid flow are very complex. Therefore, some basic assumptions are needed. Therefore, it is assumed that the water in the reservoir is non-inflatable and non-viscous flow, non-rotary and regular. Given these aspects, after a series of algebraic manipulations using the Bernoulli equation [14],[17], feed equations can be described directly int $t_{1}$, by Esq. (1) and (2).

$$
\begin{aligned}
& E_{1}=\frac{k_{m}}{A} V_{p}-\left[\frac{\alpha_{1}}{A} \sqrt{2 g}\right] \sqrt{L_{1}} \\
& E_{2}=\left[\frac{\alpha_{1}}{A} \sqrt{2 g}\right] \sqrt{L_{1}}-\left[\frac{\alpha_{2}}{A} \sqrt{2 g}\right] \sqrt{L_{2}}
\end{aligned}
$$

where $k_{m}$ represents the pump flow constant, $V_{p}$ the voltage applied to the pump, $\alpha_{i}$ the output orifice, $L_{1}, L_{2}$ the water level in $t_{1}, t_{2}$ and $g$ the gravity acceleration. In order to make the proposed system more general and perhaps conduct further studies on fault tolerance, the system is modified by introducing another pump with the same characteristic as the first one, as shown in figure 3 (b). In this case, the system has only one input and one output (SISO). Now, the equations of the multiple inputs and multiple output (MIMO) system can be described by Esq. (3) and (4)

$$
\begin{aligned}
& E_{1}=\frac{k_{m}}{A} V_{p_{1}}-\left[\frac{\alpha_{1}}{A} \sqrt{2 g}\right] \sqrt{L_{1}} \\
& E_{2}=\frac{k_{m}}{A} V_{p_{2}}+\left[\frac{\alpha_{2}}{A} \sqrt{2 g}\right] \sqrt{L_{1}}-\left[\frac{\alpha_{2}}{A} \sqrt{2 g}\right] \sqrt{L_{2}}
\end{aligned}
$$

where $V_{p_{1}}$ the voltage is applied to the first pump and $V_{p_{2}}$ is the voltage applied to the second pump.

\section{Simulation of Faults}

The various faults that present in a coupled water tanks, only some of these were selected to be simulated, as shown in Table1.

Since these types of simulation are usually exposed to adverse conditions, the proposed system simulates arithmetically.

\section{Neural Structures}

Neural Networks should be chosen for identification and FDD carefully, since they are (inefficient) and do not perform the function assigned to them. The neural structure of identification, which must represent the dynamics of the system, has a single Neural Network. . Which receives as input the past values of the levels $L_{1}(k-1), L_{2}(k-1)$, and voltages applied, $V_{p_{1}}(k-1)$ and $V_{p_{2}}(k-1)$, generating, on its output, estimated levels, called $L_{1}{ }^{\wedge}(k)$ and $L_{2}{ }^{\wedge}(k)$. The best trained Neural Network to this purpose is obtained from a second-order model NNARX (Neural Network Auto Regressive external) with eight neurons on hidden layer. The mean square error approximately is $3.73 \times 10^{-6}$. The structure of FDD, in turn, is consisted of twelve Neural Networks. In which each of these is associated with a single fault, configuring a set of "specialists". However, it is not a committee machine, since there is no network that performs the decision-making. The input of each network is consisted of the past values of the levels, $L_{1}(k-1)$ and $L_{2}(k-1)$, the voltages applied to the pumps, $V_{p_{1}}(k-1)$ and $V_{p_{2}}(k-1)$. The residual errors produced from the difference between the real and estimated output, $e_{i}(k)=L_{i}(k)-L_{i}{ }^{\wedge}$. The output of each network is a 2-bit binary word, which indicates whether the fault is being detected in $T_{1}$ and $T_{2}$ simultaneously. A schematic diagram can be shown in Fig. 4. The choice of this Neural Network structure is a commitment to several factors. Examples that can be highlighted are that more than one fault occurs at the same time in the system. In this case, if only one Neural Network is used, the FDD system should indicate each fault combination in the output [15],[18].

\section{Results and Discussion}

The first step to be taken for the identification and detection process is to obtain experimental samples for training Neural Networks under supervision using back propagation error. Therefore, the data is obtained by stimulating the system by applying Pseudo Random Binary Signals (PRBS) at a specific point of each tank and in the system error parameters and for error detection. The values are created in the interval specified by the minimum and maximum for each parameter are multiplied by the default values and applied to the form. All data are collected with a 400 steps sampling period, similar to those used in the real process. In possession of acquired values, training of Neural Networks began. All networks are trained offline, using Neural Networks tools from MATLAB, using an algorithm and prediction error of two level control system due to use Artificial Neural Network as shown in figure 5. The results obtained can be found in figures 5 to 17 . The first fault that will be simulated is gain non-calibration, which can be seen in figure 6 results. In this simulation the sensor gain is reduced to $98 \%$ of the default value. In this format, the system specified the fault only when modifying the parameter value. After this period, the fault is "compensated" by the controllers, which sent more voltage to the pump, causing the output to return to the specific point. However, the "compensation" is done improperly, because reading the sensor is a $25 \%$ error.

The system behaves in a manner similar to that in calibration and tank leaks, as shown in the figures 7 and 8 , especially for tank leak simulation, another output orifice is considered. This orifice has the same characteristics of the $\alpha_{1}, \alpha_{2}$ tanks output orifice, but has a different diameter. 
TABLE 1

\begin{tabular}{|c|c|}
\hline Fault & Name \\
\hline \multicolumn{2}{|c|}{ Sensors } \\
\hline 1 & Un calibrated offset \\
\hline 2 & Un calibrated gain \\
\hline 3 & Noise Sensitivity \\
\hline 4 & Burned Sensor \\
\hline \multicolumn{2}{|c|}{ Actuators } \\
\hline 5 & Un calibrated offset \\
\hline 6 & Un calibrated gain \\
\hline 7 & Noise Sensitivity \\
\hline 8 & Km variation \\
\hline 9 & Burned actuator \\
\hline \multicolumn{2}{|c|}{ Structural } \\
\hline 10 & Tank's Leak \\
\hline 11 & Tank's variation \\
\hline 12 & Tank's obstruction \\
\hline
\end{tabular}

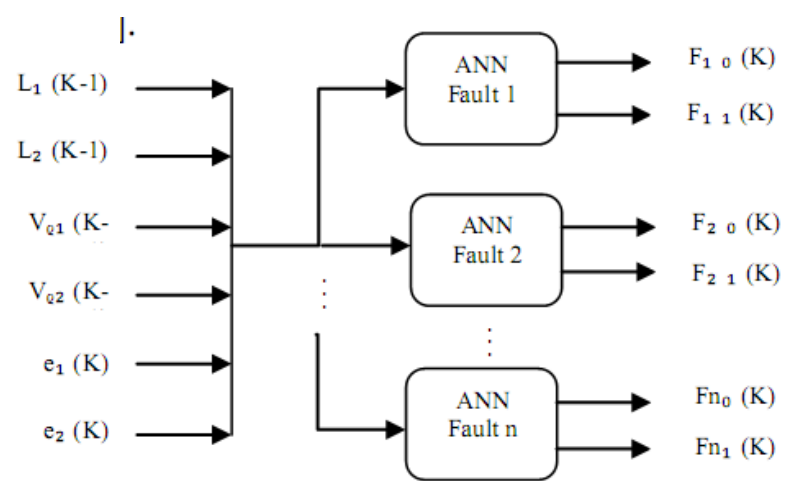

Fig. 4. Neural network structure for fault detection and diagnosis.
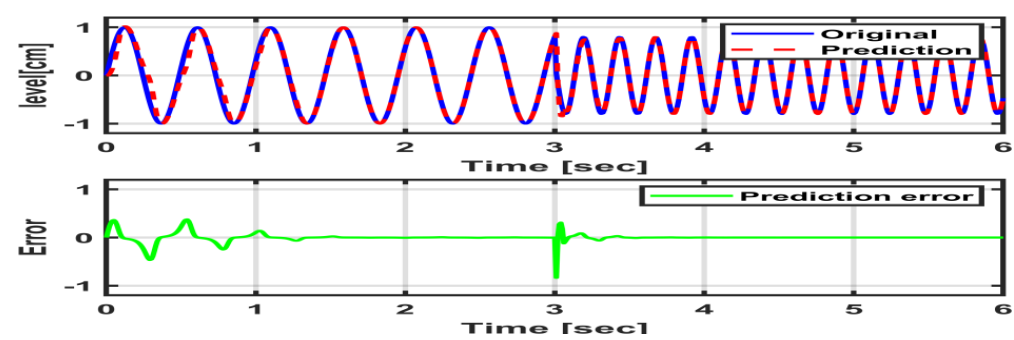

Fig.5 Training Neural Network and prediction error system.

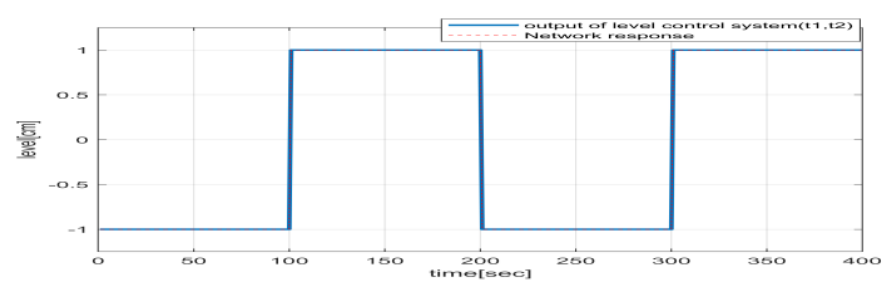

Fig. 6 Uncelebrated gain simulation-sensor gain reduced to $98 \%$ from the default value.

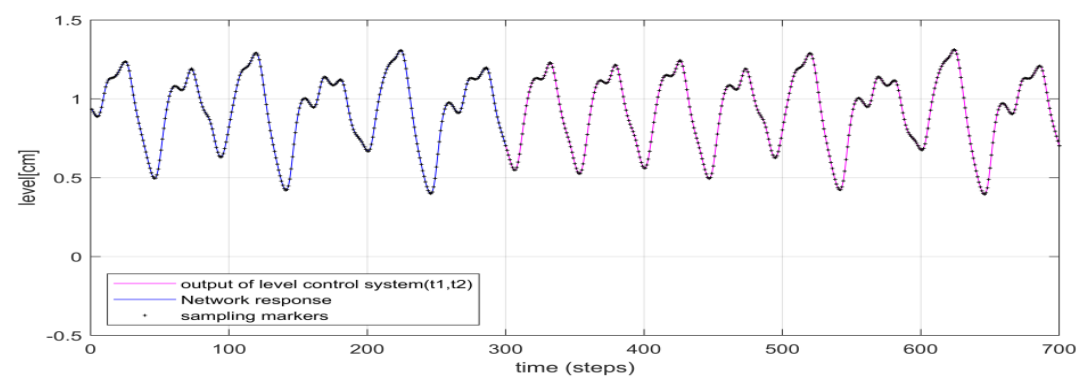

Fig. 7 uncelebrated simulation - Sensor's offset configured to- $1.5 \mathrm{~cm}$. 


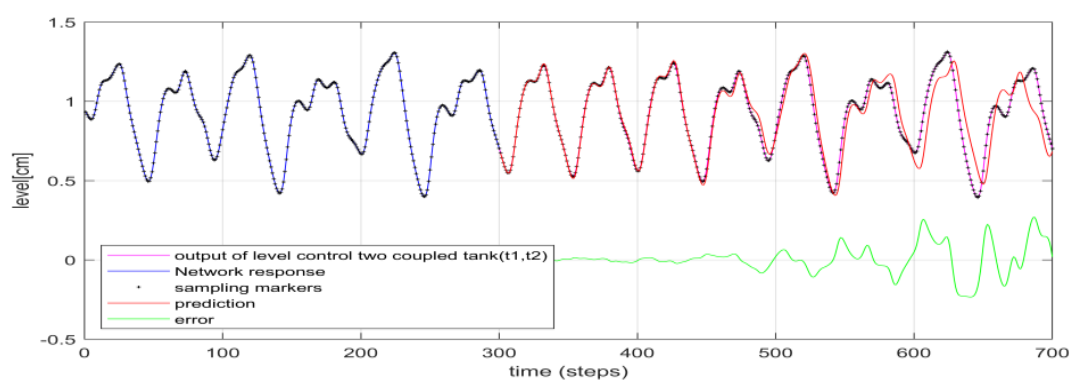

Fig. 8 Tank's Leak simulation.

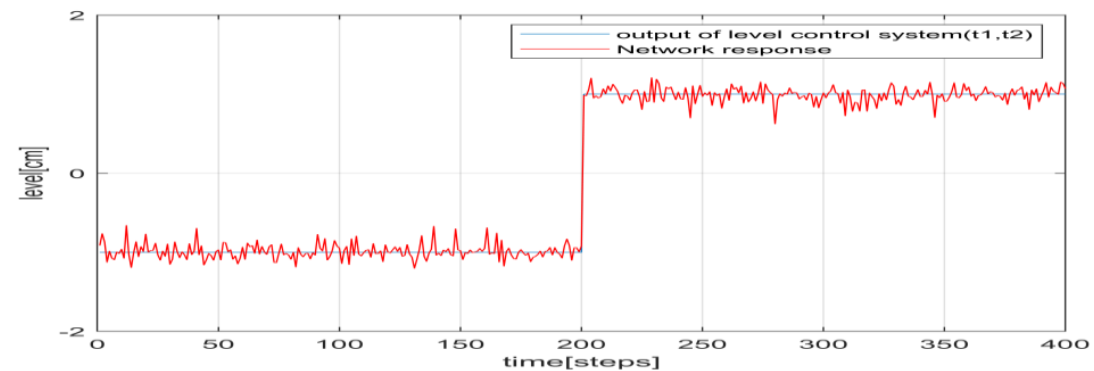

Fig. 9 The noiseity of sensors simulation - Assuming a uniform distribution noise from $\pm 2 \%$.

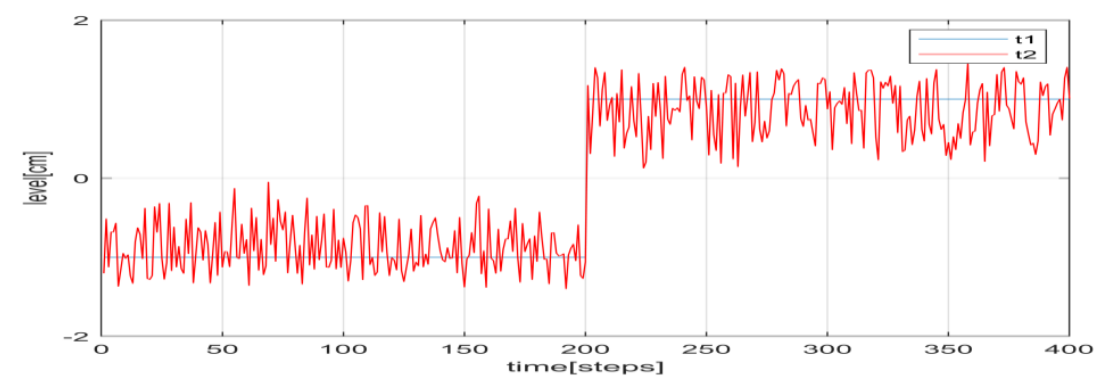

Fig. 10 Noise sensitivity simulation of actuator- Assuming a uniform distribution noise from $\pm 2 \%$.

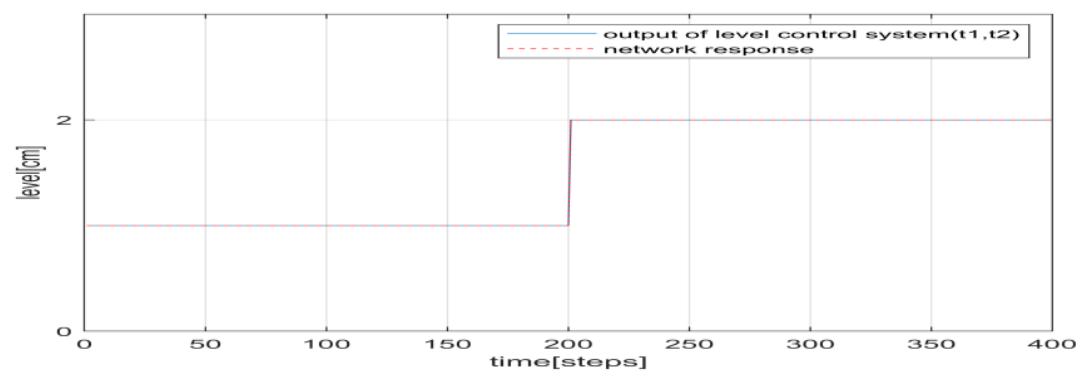

Fig. 11 Burned sensor simulation - Sensor's gain reduced to zero.

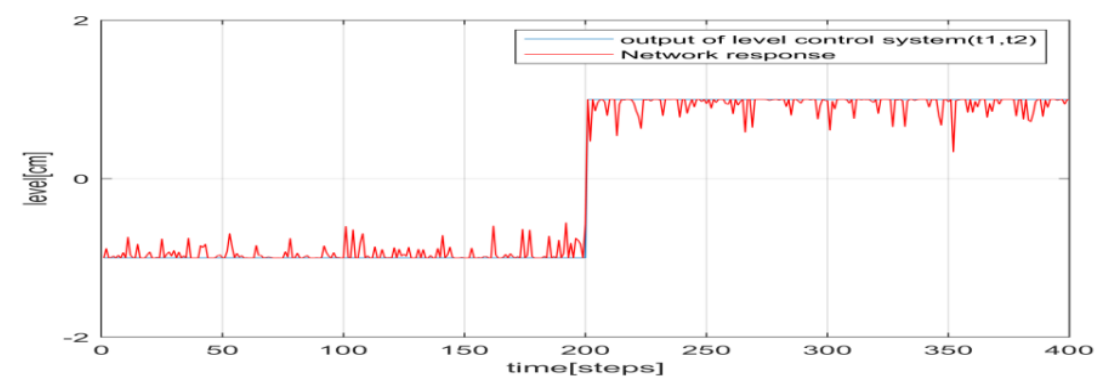

Fig. 12 Uncelebrated simulation - Actuator's gain reduced to $98 \%$ from the default value. 


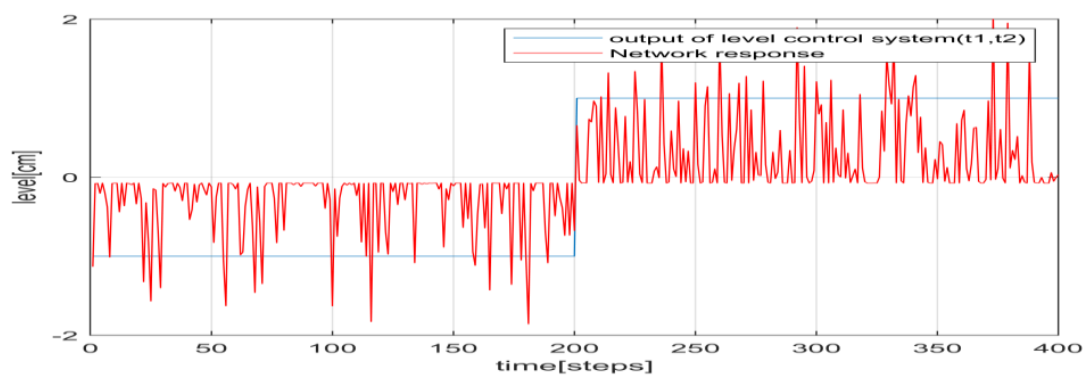

Fig. 13 Uncelebrated offset simulation - Actuator's offset configured to -1.5 volts.

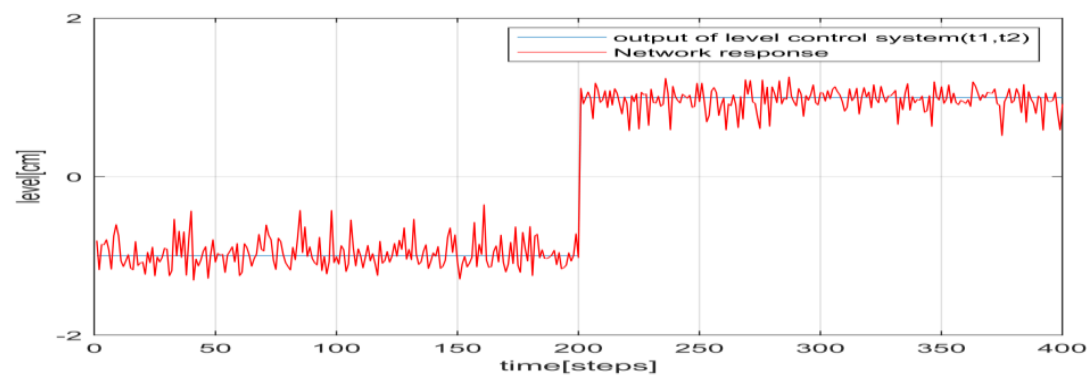

Fig. 14 Variation $-k_{m}$ reduced to $85 \%$ from the default value.

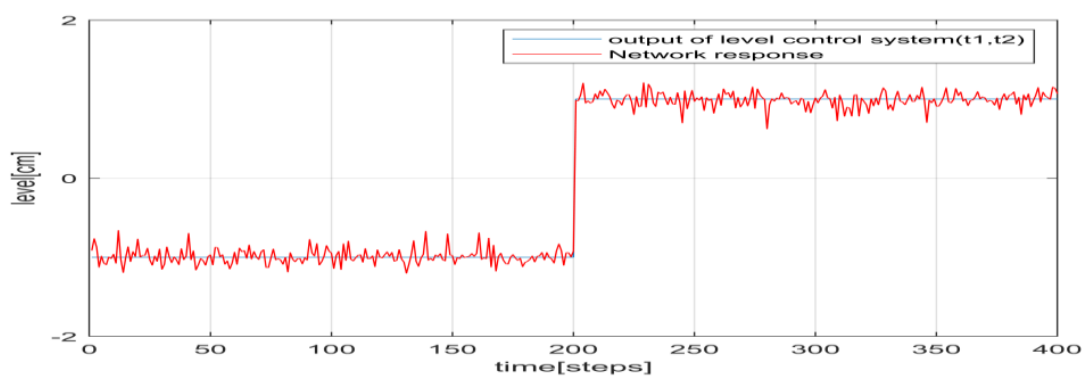

Fig. 15 Burned actuator simulation - actuator's gain reduced to zero.

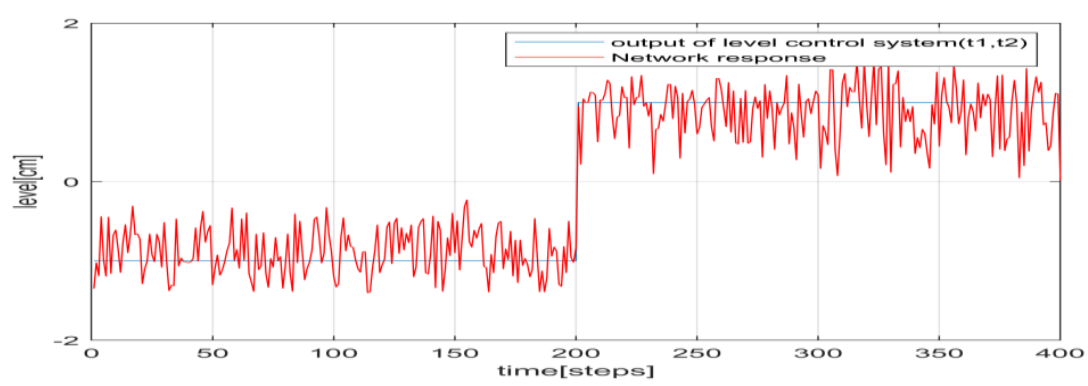

Fig. 16 Tank's $\alpha_{i}$ obstraction simulation.

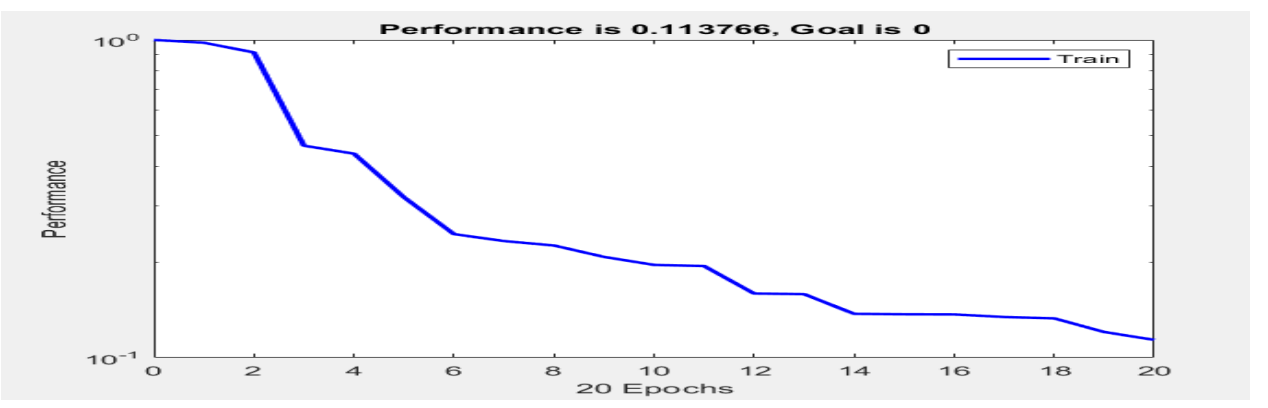

Fig. 17 Performance of the system level control. 
The noise sensitivity simulation as shown in figure 9 of the sensor is easily recognized by the system. However, because of noise with uniform distribution $( \pm 2 \%)$, the system can detect error at some points. At these points, the value generated by the RAND function keeps the signal next to the assignment point.

Unlike the noise sensitivity of the sensor, the simulation performed for the noise sensitivity of the motors is not easily recognized, as shown in figure 10 . The results obtained for $t_{1}$ can be considered as reasonable, while the results for $t_{2}$ are clearly unacceptable, since the network is not recognize any of the points where the error should have been not identified.

As well as the noise sensitivity of the sensors, all other remaining errors are also recognized by the system easily, as shown in the figures 11 to 17 .

The figure 11 is presented the simulation of the sensor when the gain of the sensor reduced to zero that is cause of burned the sensor.

In figure 12 , simulated actuator gain is reduced to $98 \%$ of the default value. In this format, the system selects the fault only when you modify the parameter value. After this period, the fault is "compensated" by the controllers, which send more voltage to the valve, resulting in the output returned to the specified point.

Finally, performance of the level control system is achieved due to train Artificial Neural Network which makes the system work more flexibly under operating conditions as shown in figure 17 . The goal is nearly to zero.

\section{Conclusion}

This work is developed in order to provide a FDD system for a coupled water tanks. Early detecting faults are very important to protect the system from collapse. In this work we have used Artificial Neural Networks to diagnose faults because they are better than the traditional methods used to diagnose faults and can deal with nonlinear systems. FDD based on ANN is achieved several advantages, maximize system reliability, survivability, maintainability, availability, cost efficiency, safety, and quality by taking full advantage of system redundancy, either in hardware or analytical form. The system uses a neural structure to process available values and inform the user of errors that occur in sensors, actuators and structural faults. Artificial Neural Networks have fully functioned in detecting system faults from other traditional control systems, maintaining system efficiency and integrity from collapse. The simulation results reflects the superiority of RNN in FDD over other networks for dynamic systems.

\section{References}

[1] R. Isermann, "Model-based fault-detection and diagnosis-status and applications", Annual Reviews in control, Vol. 29, pp. $71-$ $85,2005$.

[2] Mogens Blanke. Michel Kinnaert. Jan Lunze. Marcel Staroswiecki, "diagnosis and fault- tolerant control", $2^{\text {nd }}$ Edition, 2006, Springer.
[3] D. Himmelblau, "Fault Detection and Diagnosis in Chemical and Petrochemical Processes", Elsevier, Chemical Eng. Monograph Vol. 8, 1978.

[4] M. Witczak. "Toward the training of feed-forward neural networks with the D optimum input sequence. IEEE Transactions on Neural Networks", 17(2):357-373, 2006.

[5] J. Korbicz, J. Ko'scielny, Z. Kowalczuk, and W. Cholewa (Eds). Fault diagnosis. "Models, Artificial Intelligence, Applications", Springer-verlag, Berlin, 2004.

[6] R.J. Patton, P. M. Frank, and R. N. Clarke, "Fault diagnosis in dynamic systems: theory and applications", Prentice Hall, 1989.

[7] J. Gertler, "Fault Detection and Diagnosis in Engineering Systems “, New York: Marcel Dekker Inc., 1998.

[8] C. Edwards, T. Lombaerts, and H. Smaili, "Fault Tolerant Flight Control: A Benchmark Challenge “, vol. 399. Springer Verlag, 2010.

[9] G. Liu. D. Wang, and Y. Li, "Active fault tolerant control with actuation reconfiguration", IEEE Trans. Aerospace and Electronic Systems, Vol. 40, No. 3, pp. 1110-1117, 2004.

[10] D. Theilliol, C. Join, and Y. M. Zhang, "Actuator fault tolerant control design based on a reconfigurable reference input", Int. J. Appl. Math. Compute. Sci., Vol. 18, No. 4, pp. 553-560, 2008.

[11] J. Kim, I, Yang, D. Lee, "Control allocation based compensation for faulty blade actuator of wind turbine ", In proceedings of the $8^{\text {th }}$ IFAC Symposium on fault Detection, Supervision and Safety of Technical Processes, Mexico City, Mexico, 29-31 August 2012; pp. 355-360.

[12] S. Simani, P. Castaldi, "Active actuator fault-tolerant control of a wind turbine benchmark model”, Int. J. Robust Nonlinear Control 2014, 24, 1283-1303.

[13] Alan S. Willsky, "A survey of design methods for failure detection in dynamic systems", Automatica, vol. 12, No. 6, pp. 601-611, 1976.

[14] R.J. Patton, P. M. Frank, and R. N. Clarke, "Fault diagnosis in dynamic systems: theory and applications", Prentice Hall, 1989.

[15] Diago Leite Reboucas, Fabio Meneghetti Ugulino de Araujo, Andre Laurindo Maitelli, "USE OF ARTIFICIAL NEURLAR NETWOKS TO FAULT DETECTION AND DIAGNOSIS, ABCM Symposium Series in Mechatronics- vol. 5, 2012.

[16] Svetla Vassileva, Lyubka Doukovska, Vassil Sgurev, “AI-BASED DIAGNOSTICS FOR FAULT DETECTION AND ISOLATION IN PROCESS EQUIPMENT SERVICE", Computing and Informatics, Vol. 33,pp. 387-409,2014.

[17] Ahmad Azharuddin Azhari Mohd Amiruddin, Haslinda Zabiri, Syed A. Taqvi, Lemma Dendena Tufa, "Neural network applications in fault diagnosis and detection: an overview of implementations in engineering-related systems", ResearchGate, vol. 12, No. 6, pp. 268-275, 2018.

[18] Hye-Rin Hwang's, Berm-Soo Kim's, Tae-Hyun Cho's, In-Soo Lee's, "Implementation of a Fault Diagnosis System Using Neural Networks", International Journal of Control Automation and Systems (INT J CONTROL AUTOM), 2019. 